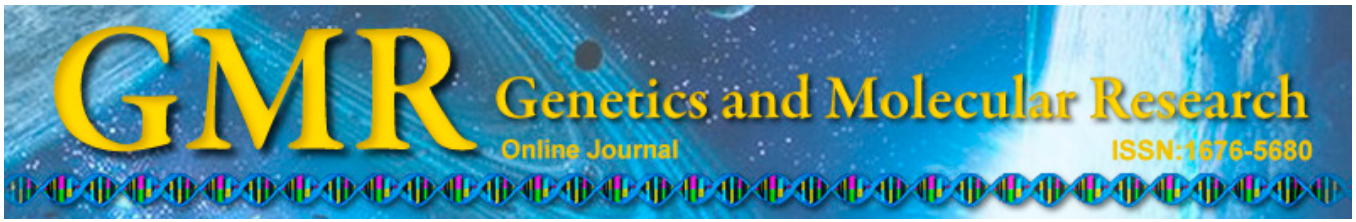

\title{
Transcriptomic identification of chemoreceptor genes in the red palm weevil Rhynchophorus ferrugineus
}

\author{
W. Yan ${ }^{1,2}$, L. Liu ${ }^{1}$, W.Q. Qin ${ }^{1}$, C.X. Li ${ }^{1}$ and Z.Q. Peng ${ }^{3}$ \\ ${ }^{1}$ Coconut Research Institute, \\ Chinese Academy of Tropical Agricultural Sciences, Hainan, China \\ ${ }^{2}$ Key Laboratory for Silviculture and Conservation of Ministry of Education, \\ Beijing Forestry University, Beijing, China \\ ${ }^{3}$ Environment and Plant Protection Institute, \\ Chinese Academy of Tropical Agricultural Sciences, Danzhou, China \\ Corresponding authors: W.Q. Qin \\ E-mail: qwq268@126.com
}

Genet. Mol. Res. 14 (3): 7469-7480 (2015)

Received October 15, 2014

Accepted April 6, 2015

Published July 3, 2015

DOI http://dx.doi.org/10.4238/2015.July.3.23

\begin{abstract}
Olfaction is crucial for insects' survival because it enables them to recognize various environmental information. It is primarily mediated by a large family of chemoreceptors, including olfactory receptors (ORs), gustatory receptors (GRs), and ionotropic receptors (IRs). Here, we assembled the transcriptome of the economically important pest of palms, Rhynchophorus ferrugineus, to reveal its chemoreceptor gene repertoire. About $8.08 \mathrm{Gbp}$ data were generated using a HiSeq platform and their assembly led to a total of 24,439 unigenes. Among the transcripts, 12,523 (51.24\%) showed significant similarity (E-value $<10^{-5}$ ) to known proteins in the National Center for Biotechnology Information $\mathrm{Nr}$ database. From these sequences, 18 candidate genes of ORs were identified. Nine putative transcripts were homologous to GR genes, while 9 were similar to IR genes. The expression profiles of all identified chemoreceptor genes were
\end{abstract}


determined by quantitative real-time PCR in antenna, head, thorax, abdomen, and legs of both sexes. Most chemoreceptor genes were antenna-enriched. This study demonstrated a successful application of a transcriptome for discovering a large number of divergent chemoreceptor genes of a non-model organism. The findings provide a valuable sequence resource and gene tissue distribution information for systematic functional analysis of molecular mechanisms underlying chemoreception in this pest.

Key words: Rhynchophorus ferrugineus; Expression pattern; Transcriptome; Chemoreception

\section{INTRODUCTION}

The red palm weevil Rhynchophorus ferrugineus (Coleoptera: Dryophthoridae), native to tropical Asian regions, is the most threatening pest in palms worldwide. Currently, large-scale $R$. ferrugineus outbreaks have resulted in unprecedented economic losses (Avalos et al., 2014). R. ferrugineus conceals itself by boring into palm tissues, which is one of the main obstacles to its efficient control. Despite a wide range of measures used to prevent and control R. ferrugineus infestations (Faleiro and Ashok Kumar, 2008), the current method is mainly based on the application of large quantities of insecticides in order to limit the spread of infestation (Llácer et al., 2013).

The olfactory sense drives beetle behaviors that are of utmost important for fitness, such as the localization of suitable hosts and mates (Andersson et al., 2013). Based on information chemically communicated by adults, traps baited with aggregation pheromones and date fruits have been developed as effective measures to control $R$. ferrugineus (Abraham et al., 1999; Abuagla et al., 2012). A better knowledge of the molecular mechanism of the olfaction of this pest will offer the possibility to develop other olfactory-based strategies to disrupt critical behaviors, such as sex pheromone-mediated reproduction, host selection, and oviposition (Glaser et al., 2013). Olfaction is a complex process requiring the interaction of numerous proteins to generate a neuronal signal. The large families of chemoreceptors that evolved through repeated processes of gene duplication and diversification are important for olfactory recognition, which is formed by the olfactory receptors (ORs), gustatory receptors (GRs), and ionotropic receptors (IRs) located in the dendritic membrane of neurons (Vieira and Rozas, 2011).

Despite the economic importance of $R$. ferrugineus, information on the molecular aspects of olfaction has been lacking until now. With the advantages of next-generation sequencing technologies (Zhu et al., 2013), we here reveal the chemoreceptor genes for further investigation regarding the genomics and neurobiology that underlie olfactory behavior in this devastating pest. Their gender expression patterns in different tissues were examined.

\section{MATERIAL AND METHODS}

\section{Insects}

R. ferrugineus was derived from cocoons originally collected in naturally infested palms in the suburbs of Hainan Province. The cocoons were individually placed in plastic 
containers with perforated lids and incubated until adult emergence. After emergence, adults were bred in pairs using sugarcane as both a food and oviposition substrate. Eggs were collected from sugarcane and placed on the artificial diet for hatching. The larvae were raised on an artificial diet from hatching to the ultimate instars in an environmental chamber in the dark at $25^{\circ} \mathrm{C}$ with $65 \%$ humidity (Barranco et al., 1997). Upon eclosion, adults were maintained in a holding cage under environmental conditions.

\section{cDNA library construction and sequencing}

RNA from five respective female and male adult beetles was extracted using Trizolreagent (Invitrogen, Carlsbad, CA, USA) according to manufacturer instructions. The integrity of RNA was confirmed by $1 \%$ agarose gel electrophoresis. It was then quantified using an Agilent 2100 Bioanalyzer (Agilent, Santa Clara, CA, USA). Poly (A) mRNA was isolated using oligo(dT) magnetic beads and interrupted into short fragments by fragmentation buffer. First-strand cDNA was generated using random hexamer-primed reverse transcription, followed by synthesis of the second-strand cDNA. The short fragments were connected with sequencing adaptors. These products were purified and enriched by PCR to create the final cDNA library. The library was sequenced using an Illumina HiSeq ${ }^{\mathrm{TM}} 2000$ platform.

\section{Assembly and bioinformatic analysis}

The dirty reads, including reads with adaptors, unknown nucleotides larger than 5\%, and reads of low quality (the number of bases with quality value $\leq 10$ in more than $20 \%$ ) were discarded following stringent filtering of raw sequencing reads. The clean reads were assembled using the Trinity software (an efficient de novo transcriptome assembler, particularly in the absence of a reference genome) with default settings except K-mer value used to construct unique consensus sequences (Grabherr et al., 2011). First, contigs were generated by combing the clean reads of a certain overlap length to form longer fragments. Then, unigenes were obtained by connecting the contigs into sequences that could not be extended on either end. For assignments of predicted gene descriptions, all unigenes were used for similarity searches against $\mathrm{Nr}$ and SwissProt databases with an E-value $<10^{-5}$. The best hit was used to determine the sequence direction and coding sequences of transcripts, and the peptide sequences were translated using standard codons. All eukaryotic protein domains in the unigenes were searched using the Pfam software. Functional annotation by Gene Ontology (GO), Cluster of Orthologous Groups (COG), and Kyoto Encyclopedia of Genes and Genome (KEGG) were determined using the Blast2GO software (Conesa et al., 2005). For gene expression analysis, the number of unique reads was calculated and then normalized to give the reads per kb pairs per million mapped reads (RPKM) (Mortazavi et al., 2008).

\section{Identification of chemoreceptor genes}

The $R$. ferrugineus database was queried using available OR, GR, and IR sequences from Tribolium castaneum (Vieira and Rozas, 2011) by BLASTx with an E-value $<10^{-5}$. All $R$. ferrugineus ORs, GRs, and IRs identified in this manner were used in successive BLASTx searches to identify other candidate sequences. Putative alternative splice variants were filtered based on sequence similarity. The longest protein isoform was retained for each gene. 


\section{Quantitative real time PCR (qPCR)}

Antennae, heads (without antennae), thoraxes, abdomens, and legs collected from female and male adults were dissected. Total RNA was extracted as described above. For each sample, first-strand cDNAs were reverse-transcribed from $0.5 \mu \mathrm{g}$ total RNAs treated with $4 \mathrm{X}$ gDNA wiper Mix using HiScript ${ }^{\mathrm{TM}}$ Q RT SuperMix for qPCR (Vazyme, Nanjing, Jiangsu, China) according to manufacturer instructions. About $100 \mathrm{ng}$ first-strand cDNAs for each sample were used as template for qPCR anslysis. qPCR was implemented using the SYBR premix Ex Taq kit (Takara, China) using a qPCR System (Roche, USA). Reactions were carried out at $94^{\circ} \mathrm{C}$ for $2 \mathrm{~min}$, followed by 45 cycles of $5 \mathrm{~s}$ at $94^{\circ} \mathrm{C}$ and $30 \mathrm{~s}$ at $60^{\circ} \mathrm{C}$. Amplification of an $18 \mathrm{~S}$ ribosomal RNA fragment was used as an endogenous control. The primers employed in qPCR are listed in Table S1. For each gene, the experiments were repeated for three biological replicates. Expression levels were calculated relative to the reference genes using the comparative $2^{-\Delta \Delta \mathrm{Ct}}$ method (Livak and Schmittgen, 2001). The total expression in all the tissues of one gene was set as $100 \%$. The percentage of one gene in each tissue was used to measure the expression level.

\section{Data deposition}

Raw sequence data were deposited in the DNA Data Bank of Japan Sequence Read Archive (DRA) (http://trace.ddbj.nig.ac.jp/dra/index_e.html) database with accession No. DRA002418. Assembled unigenes will be available upon request from Wei Yan (andy206@126.com).

\section{RESULTS}

\section{Sequencing and assembly}

Using Illumina paired-end sequencing technology, a total of 82,940,054 raw reads were obtained. After removing adaptor sequences, empty reads, and low quality sequences, $80,803,668$ clean reads encompassing about $8.08 \mathrm{~Gb}$ nucleotides were generated. The GC content of the sequences was $41.98 \%$. Average read size and Q20 percentage were 90 bp and $98.01 \%$, respectively. The clean reads were assembled into 42,195 contigs with an average length of $1818 \mathrm{bp}$ and an N50 of $3379 \mathrm{bp}$ (Table 1). From these, 24,439 unigenes were obtained with a mean length of $1146 \mathrm{bp}$ and an N50 of $2206 \mathrm{bp}$. The unigenes with a length over $500 \mathrm{bp}$ accounted for $51.97 \%$ of the total.

\begin{tabular}{|c|c|c|}
\hline & Contigs & Unigenes \\
\hline $200-500 \mathrm{bp}$ & 14,135 & 11,739 \\
\hline $501-1,000 \mathrm{bp}$ & 6,563 & 4,159 \\
\hline $1,001-2,000 \mathrm{bp}$ & 8,292 & 8,356 \\
\hline$>2,000 \mathrm{bp}$ & 13,205 & 4,185 \\
\hline Total number & 42,195 & 24,439 \\
\hline Total length (bp) & $76,691,858$ & $28,001,567$ \\
\hline Minimum length (bp) & 201 & 201 \\
\hline Mean length (bp) & 1,818 & 1,146 \\
\hline Median length (bp) & 1,042 & 534 \\
\hline Maximum length (bp) & 27,356 & 27,356 \\
\hline N50 (bp) & 3,379 & 2,206 \\
\hline N90 (bp) & 851 & 407 \\
\hline
\end{tabular}




\section{Functional annotation of assembled unigenes}

In total, 13,488 unigenes were annotated in at least one database of Nr, Nt, Swissport, GO, COG, KEGG, and Pfam, covering 55.19\% of all unigenes (Tables 2 and Table S2). As expected, only $3.16 \%$ unigenes were annotated in all databases mentioned above. Using BLASTx, 12,523 unigenes $(51.24 \%)$ had significant similarity to protein accessions in the Nr protein database. For functional classification, 10,334 unigenes (42.28\%) with BLAST matches to known proteins were assigned to GO classes with 188,689 functional terms and classified into three ontologies of molecular function, cellular component, and biological process (Figure 1). Of these, assignments to the biological process made up the majority $(123,974 ; 65.70 \%)$, followed by cellular component $(40,736 ; 21.59 \%)$ and molecular function $(23,979 ; 12.71 \%)$. Using COG functional annotation, 7,490 unigenes (30.64\%) were assigned to 26 function classes (Figure 2). In total, 1,463 unigenes (17.32\%) were assigned to "General function prediction only" representing the largest group, followed by "Signal Transduction" (1,136; 13.45\%), "Post-translational modification, protein turnover, chaperon" (752; $8.90 \%)$, "Function unknown" (483;5.72\%) and "Translation" (446;5.28\%). Pathway-based analyses revealed that 3,367 unigenes (13.77\%) had significant matches in the database. They were assigned to $237 \mathrm{KEGG}$ pathways. The four main classifications included cellular processes, environmental information processing, genetic information processing, and metabolism and organismal systems (Figure 3). There were 47 pathways with at least 50 assigned unigenes. The top four most prominent pathways were "Ribosome" (186; 2.47\%), "Protein processing in endoplasmic reticulum" (132; $1.76 \%)$, "Spliceosome" (123; 1.64\%), and "Lysosome" (123; 1.64\%).

Table 2. Summary of annotations of unigenes.

\begin{tabular}{lcr}
\hline Annotated databases & Number of unigenes & Percentage $(\%)$ \\
\hline $\mathrm{Nr}$ & 12,523 & 51.24 \\
$\mathrm{Nt}$ & 2,106 & 8.61 \\
SwissProt & 9,831 & 40.22 \\
PFAM & 9,907 & 40.53 \\
GO & 10,334 & 42.28 \\
$\mathrm{COG}$ & 3,367 & 13.77 \\
KEGG & 7,490 & 30.64 \\
All databases & 773 & 3.16 \\
At least one database & 13,488 & 55.19 \\
Total & 24,439 & 100.00 \\
\hline
\end{tabular}

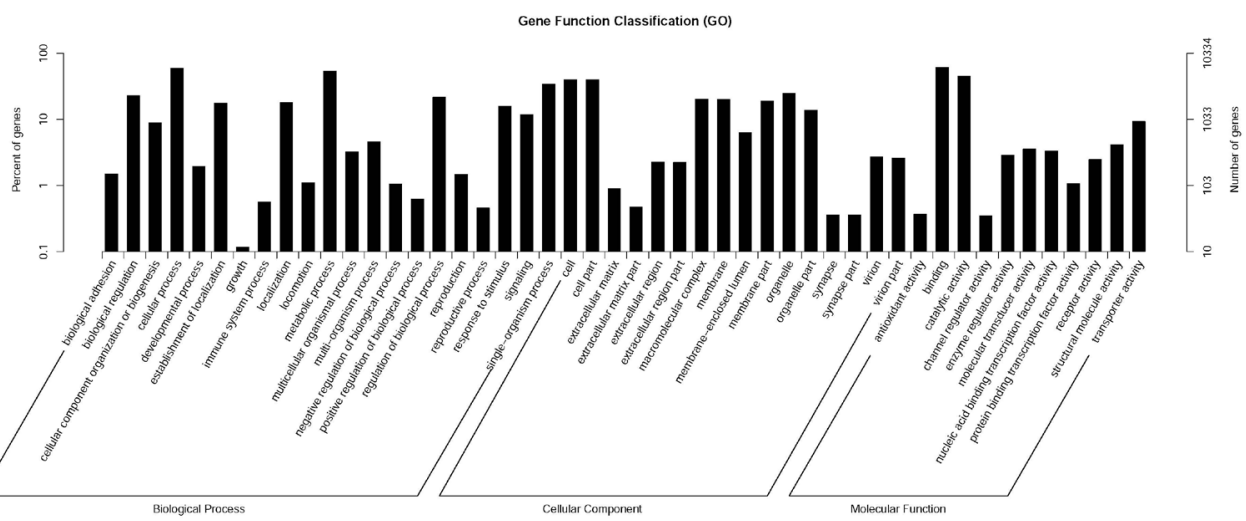

Figure 1. Description of the three categories of Gene Ontology (GO) terms for the transcriptomic sequences. 
KOG Classification

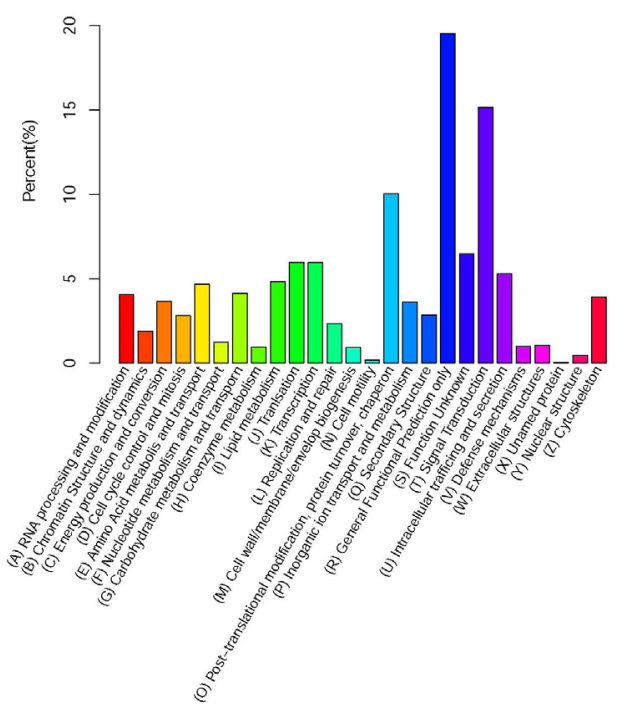

Figure 2. Clusters of orthologous groups (COG) predicted from the unigenes.

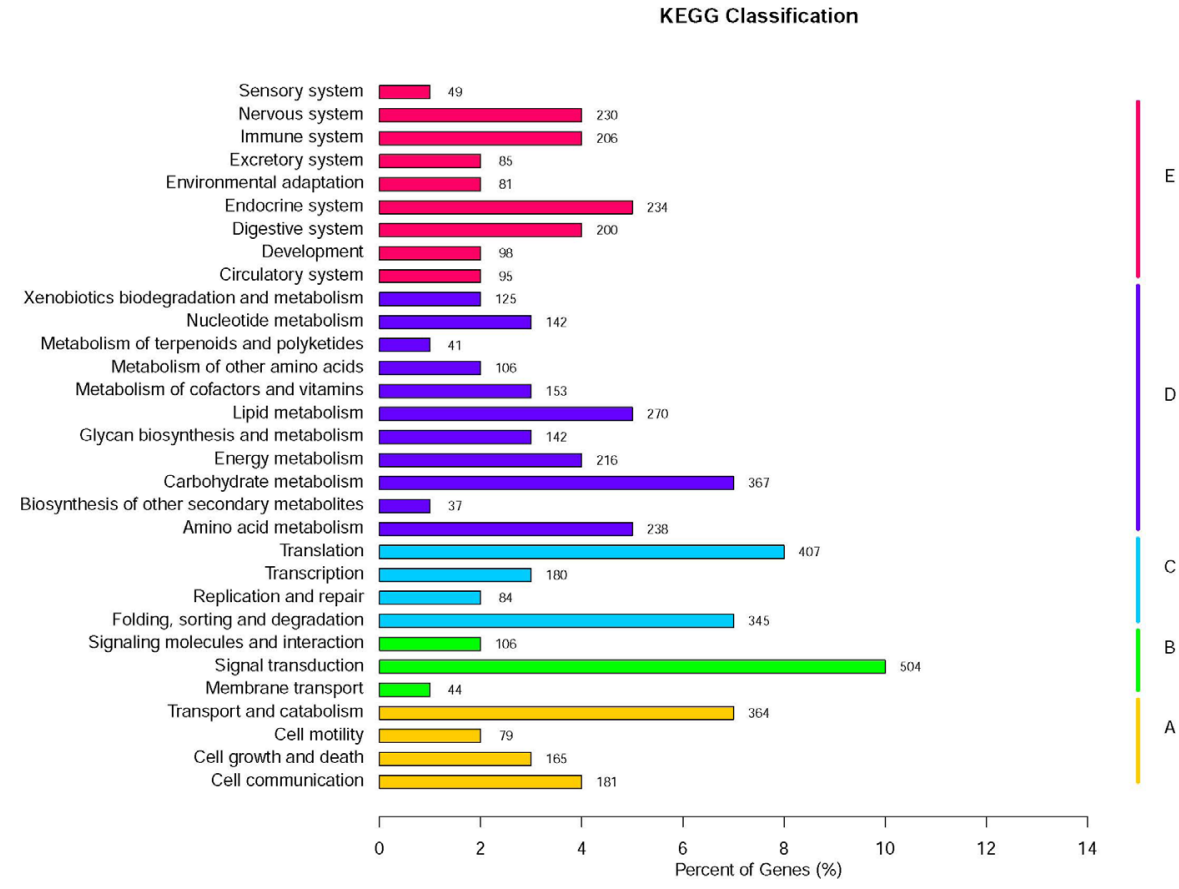

Figure 3. Summary of KEGG pathway annotation of the transcriptome. A. Cellular processes. B. Environmental information processing. C. Genetic information processing. D. Metabolism. E. Organismal systems. 


\section{Putative chemoreceptor genes}

A homologous method was performed to identify chemoreceptor genes. In $R$. ferrugineus sequences, 36 putative chemoreceptor genes were identified (Table 3 ). All these transcripts possessed overlapping regions with low identity and therefore represented unigenes. Interestingly, almost all of these were blasted against homologs of T. castaneum and Dendroctonus ponderosae. Only two genes (comp504_c0 and comp186697_c0) appeared to be closer to homologs of Drosophila grimshawi and Bombyx mori, respectively. There were 18 ORs, 9 GRs and 9 IRs. Most of them were partial gene sequences. Only comp18004_c0, comp6569 c0 and comp15615 c0 were found to represent full-length open reading frames. Among OR genes, comp18004_c0, comp6569 c0, comp15615 c0 and comp6619 c0 had high levels of expression with an RPKM of 17.81, 18.00, 7.97, and 27.14, respectively. All other transcripts had low expression levels below 5 RPKM. Among GR genes, the relative high-level expressions were detected for comp10251_c0, comp17518_c0 and comp14468_c0 with an RPKM of 3.71, 2.31, and 2.09, respectively. All IR genes had low expression levels less than 1 RPKM.

\begin{tabular}{|c|c|c|c|c|c|c|}
\hline \multirow[t]{2}{*}{ Unigene ID } & \multirow[t]{2}{*}{ Length (bp) } & \multirow[t]{2}{*}{ RPKM } & \multicolumn{4}{|c|}{ BLASTx best hit } \\
\hline & & & Accession No. & Score & E-value & Species \\
\hline 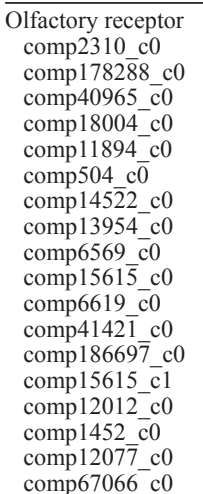 & $\begin{array}{r}826 \\
239 \\
528 \\
5494 \\
850 \\
425 \\
836 \\
1057 \\
1656 \\
2087 \\
1130 \\
356 \\
676 \\
242 \\
1206 \\
986 \\
447 \\
1511\end{array}$ & $\begin{array}{r}1.35 \\
0.34 \\
2.52 \\
27.81 \\
1.47 \\
0.64 \\
2.34 \\
3.88 \\
18.00 \\
7.97 \\
27.14 \\
3.28 \\
0.56 \\
0.11 \\
1.37 \\
1.60 \\
1.21 \\
1.17\end{array}$ & $\begin{array}{l}\text { AFI45064 } \\
\text { AEE61493 } \\
\text { AEE61404 } \\
\text { EFA06217 } \\
\text { AFI45064 } \\
\text { XP } 001986725 \\
\text { EFA01342 } \\
\text { AEE63155 } \\
\text { XP } 974059 \\
\text { EFA06088 } \\
\text { EFA11578 } \\
\text { AEE61404 } \\
\text { NP 001104798 } \\
\text { XP966790 } \\
\text { AEE61493 } \\
\text { AEE62637 } \\
\text { EEZ99171 } \\
\text { EFA10800 }\end{array}$ & $\begin{array}{r}947 \\
188 \\
284 \\
3189 \\
1206 \\
121 \\
135 \\
170 \\
1735 \\
683 \\
219 \\
179 \\
180 \\
156 \\
612 \\
587 \\
269 \\
450\end{array}$ & $\begin{array}{l}6.26 \mathrm{E}-123 \\
1.65 \mathrm{E}-15 \\
1.52 \mathrm{E}-27 \\
0 \\
8.76 \mathrm{E}-162 \\
6.76 \mathrm{E}-06 \\
9.03 \mathrm{E}-07 \\
9.36 \mathrm{E}-11 \\
0 \\
4.07 \mathrm{E}-79 \\
1.87 \mathrm{E}-19 \\
1.10 \mathrm{E}-13 \\
6.42 \mathrm{E}-13 \\
4.55 \mathrm{E}-11 \\
6.21 \mathrm{E}-72 \\
3.28 \mathrm{E}-69 \\
7.54 \mathrm{E}-26 \\
1.30 \mathrm{E}-47\end{array}$ & $\begin{array}{l}\text { Dpo } \\
\text { Dpo } \\
\text { Dpo } \\
\text { Tca } \\
\text { Dpo } \\
\text { Dgr } \\
\text { Tca } \\
\text { Dpo } \\
\text { Tca } \\
\text { Tca } \\
\text { Tca } \\
\text { Dpo } \\
\text { Bmo } \\
\text { Tca } \\
\text { Dpo } \\
\text { Dpo } \\
\text { Tca } \\
\text { Tca }\end{array}$ \\
\hline 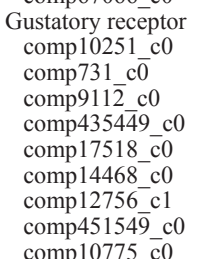 & $\begin{array}{r}439 \\
728 \\
1388 \\
221 \\
1220 \\
1211 \\
549 \\
261 \\
205\end{array}$ & $\begin{array}{l}3.71 \\
0.67 \\
1.70 \\
0.12 \\
2.31 \\
2.09 \\
1.53 \\
0.31 \\
0.40\end{array}$ & $\begin{array}{l}\text { CAL23188 } \\
\text { EEZ99385 } \\
\text { NP } 001138948 \\
\text { CALL23162 } \\
\text { CAL23187 } \\
\text { XP } 968269 \\
\text { EFĀ11612 } \\
\text { XP } 001813249 \\
\text { EFĀ04717 }\end{array}$ & $\begin{array}{r}193 \\
134 \\
1032 \\
144 \\
215 \\
215 \\
269 \\
143 \\
124\end{array}$ & $\begin{array}{l}2.86 \mathrm{E}-15 \\
7.40 \mathrm{E}-07 \\
5.87 \mathrm{E}-134 \\
1.66 \mathrm{E}-09 \\
8.13 \mathrm{E}-17 \\
2.09 \mathrm{E}-16 \\
1.97 \mathrm{E}-25 \\
8.52 \mathrm{E}-10 \\
7.18 \mathrm{E}-07\end{array}$ & $\begin{array}{l}\text { Tca } \\
\text { Tca } \\
\text { Tca } \\
\text { Tca } \\
\text { Tca } \\
\text { Tca } \\
\text { Tca } \\
\text { Tca } \\
\text { Tca }\end{array}$ \\
\hline $\begin{array}{l}\text { Ionotropic receptor } \\
\text { comp3118c0 } \\
\text { comp243668_c0 } \\
\text { comp369944_c0 } \\
\text { comp215061_c0 } \\
\text { comp264607_c0 } \\
\text { comp14614_c1 } \\
\text { comp10726_c1 } \\
\text { comp3333 c0 } \\
\text { comp10889_c0 }\end{array}$ & $\begin{array}{l}365 \\
311 \\
224 \\
231 \\
237 \\
340 \\
668 \\
336 \\
207\end{array}$ & $\begin{array}{l}0.52 \\
0.35 \\
0.12 \\
0.47 \\
0.23 \\
0.40 \\
0.93 \\
0.65 \\
0.12\end{array}$ & $\begin{array}{l}\text { XP-975120 } \\
\text { XP-968638 } \\
\text { XP-968346 } \\
\text { XP-971258 } \\
\text { XP-968638 } \\
\text { XP-971258 } \\
\text { XP-974691 } \\
\text { EEZ̄99899 } \\
\text { XP_968638 }\end{array}$ & $\begin{array}{l}405 \\
196 \\
343 \\
251 \\
140 \\
590 \\
723 \\
512 \\
215\end{array}$ & $\begin{array}{l}1.50 \mathrm{E}-44 \\
3.91 \mathrm{E}-16 \\
2.68 \mathrm{E}-36 \\
1.57 \mathrm{E}-23 \\
7.79 \mathrm{E}-09 \\
1.72 \mathrm{E}-69 \\
1.05 \mathrm{E}-86 \\
9.04 \mathrm{E}-59 \\
3.58 \mathrm{E}-19\end{array}$ & $\begin{array}{l}\text { Tca } \\
\text { Tca } \\
\text { Tca } \\
\text { Tca } \\
\text { Tca } \\
\text { Tca } \\
\text { Tca } \\
\text { Tca } \\
\text { Tca }\end{array}$ \\
\hline
\end{tabular}

$\mathrm{RPKM}=$ reads per $\mathrm{kb}$ pairs per million mapped reads. $\mathrm{Bmo}=$ Bombyx mori $; \mathrm{Dpo}=$ Dendroctonus ponderosae; $\mathrm{Dgr}=$ Drosophila grimshawi $; \mathrm{Tca}=$ Tribolium castaneum . 


\section{Tissue-specific expression patterns}

qPCR analyses were conducted to assess the expression of identified putative chemoreceptor genes in different tissues including antennae, heads (without antennae), thoraxes, abdomens, and legs. The expressions of all the 36 identified chemoreceptor genes were detected in antennae (Table 4). However, comp14522_c0, comp12012_c0, comp1452_c0, comp731_c0, comp17518_c0, and comp10889_c0 had low antennal expression levels as compared with that of other tissues. In antennae, the expression of genes tested was higher in males than in females. Among the OR genes, comp2310_c0, comp178288_c0, comp40965 c0, comp11894_c0, comp504_c0, comp15615_c1, and comp67066_c0 were highly specific to antennae. The expression of comp14522_c0 and comp12012_c0 was enriched in the female abdomen. Similarly, high expression in the male abdomen was observed for comp504_c0 and comp13954_c0. Expression levels of comp41421_c0 and comp1452_c0 in tissues other than female legs were uniformly low. Of the GR genes, the transcript levels of comp14468_c0, comp451549_c0, and comp10775_c0 were much higher in both male and female antennae than in other tissues. The transcript of comp10251_c0 was expressed at its high levels in male antennae followed by heads and legs, while its expression level in female antennae was lower than that of these three male tissues. Expression of comp17518 c0 was enriched exclusively in female heads. Expression of comp731_c0 and comp12756_c 1 in the female abdomen was much higher than in other tissues of both females and males. Comp9112_c0 was not only highly expressed in male antennae but also in the female abdomen. With respect to IR genes, all expect for comp264607_c0 and comp10889_c0 were significantly enriched in male antennae as compared with female antennae. Comp 264607 c0 was expressed at its highest level in male antennae followed by female thoraxes and heads. High expression of comp10889_c0 was comparably detected in female legs and the male abdomen.

\section{DISCUSSION}

Because the chemoreceptor genes are divergent with low sequence identity and olfactory tissues controlled by genes with restricted expression are difficult to collect, it is very difficult to identify a large number of chemoreceptor genes by traditional molecular cloning and cDNA library sequencing strategies (Zhu et al., 2012). Although a large number of chemoreceptor genes have been deposited, most of them were identified from species with complete genomes determined by bioinformatic approaches (Sánchez-Gracia et al., 2009). Using the powerful and cost-effective advantages of next-generation sequencing for obtaining functional genes in non-model organisms, we identified 36 chemoreceptor genes in the $R$. ferrugineus transcriptome. These results indicate that transcriptome sequencing is an efficient approach for identifying a large array of divergent chemoreceptor genes in a species with no genomic data available. This method has been recently verified in a few insect species, including D. ponderosae, Ips typographus, Nilaparvata lugens, Spodoptera littoralis, and Spalangia endius (Andersson et al., 2013; Poivet et al., 2013; Zhou et al., 2013; Zhang et al., 2014). Compared to those found in other insects, detailed genomic analysis revealed a large number of chemoreceptors in T. castaneum (Engsontia et al., 2008). Although the gene number of chemoreceptors varied in different orders or species, and the number of chemoreceptors in $R$. ferrugineus is within the range identified in other insect species (Sánchez-Gracia et al., 2009), additional chemoreceptors may await discovery due to their absence from the current 
Table 4. Tissue-specific expression profiles of putative chemoreceptor genes.

\begin{tabular}{|c|c|c|c|c|c|c|c|c|c|c|}
\hline \multirow[t]{2}{*}{ Gene } & \multicolumn{5}{|c|}{ Female (\%) } & \multicolumn{5}{|c|}{ Male $(\%)$} \\
\hline & Head & Thorax & Abdomen & Leg & Antennae & Head & Thorax & Abdomen & Leg & Antennae \\
\hline \multicolumn{11}{|l|}{ Olfactory receptor } \\
\hline comp178288_c0 & 0.01 & 0.02 & 0.32 & 0.00 & 16.54 & 0.26 & 0.18 & 0.08 & 0.03 & 82.55 \\
\hline comp40965_c0 & 0.30 & 0.55 & 2.29 & 0.55 & 12.42 & 0.47 & 0.24 & 14.24 & 0.56 & 68.38 \\
\hline comp18004_c0 & 8.02 & 9.23 & 9.20 & 5.66 & 7.91 & 9.00 & 4.76 & 3.48 & 5.91 & 36.82 \\
\hline comp11894_c0 & 0.01 & 0.02 & 0.00 & 0.40 & 27.45 & 0.00 & 0.00 & 0.00 & 0.01 & 72.10 \\
\hline comp504_c0 & 0.00 & 0.00 & 0.02 & 0.00 & 16.22 & 0.00 & 0.00 & 38.65 & 0.00 & 45.11 \\
\hline comp14522_c0 & 0.42 & 0.70 & 95.27 & 0.00 & 0.46 & 0.53 & 0.39 & 0.00 & 0.00 & 2.23 \\
\hline comp13954_c0 & 33.41 & 0.17 & 0.12 & 0.18 & 7.91 & 0.00 & 0.00 & 33.89 & 0.31 & 24.02 \\
\hline comp6569_c0 & 7.08 & 9.43 & 13.52 & 5.26 & 11.24 & 6.16 & 4.89 & 3.43 & 7.20 & 31.79 \\
\hline comp15615_c0 & 1.53 & 1.18 & 0.61 & 1.80 & 18.55 & 1.34 & 0.74 & 9.34 & 1.74 & 63.16 \\
\hline comp6619_c0 & 2.99 & 2.47 & 2.24 & 2.63 & 13.05 & 3.79 & 2.42 & 7.73 & 2.17 & 60.51 \\
\hline comp41421_c0 & 4.20 & 1.79 & 9.86 & 59.36 & 4.66 & 5.98 & 1.81 & 0.27 & 2.27 & 9.80 \\
\hline comp186697_c0 & 4.48 & 3.08 & 15.33 & 1.05 & 0.87 & 2.99 & 0.35 & 1.33 & 36.39 & 34.14 \\
\hline comp15615_c1 & 1.67 & 2.13 & 0.98 & 3.38 & 25.17 & 1.68 & 0.79 & 0.39 & 1.68 & 62.15 \\
\hline comp12012_c0 & 0.78 & 1.27 & 47.50 & 0.70 & 1.10 & 16.53 & 15.28 & 11.88 & 2.87 & 2.09 \\
\hline comp1452_c0 & 0.30 & 0.53 & 1.30 & 79.72 & 4.79 & 0.33 & 0.03 & 0.60 & 4.89 & 7.51 \\
\hline comp12077_c0 & 0.19 & 2.44 & 11.92 & 0.08 & 10.97 & 0.25 & 0.61 & 5.08 & 0.22 & 68.24 \\
\hline comp67066_c0 & 4.84 & 4.35 & 5.22 & 2.86 & 24.40 & 8.32 & 2.65 & 1.56 & 4.02 & 41.78 \\
\hline \multicolumn{11}{|l|}{ Gustatory receptor } \\
\hline comp10251_c0 & 8.05 & 5.88 & 4.48 & 6.93 & 8.36 & 14.17 & 7.08 & 2.54 & 11.93 & 30.59 \\
\hline comp731_cc 0 & 0.44 & 10.51 & 53.07 & 0.85 & 1.19 & 0.29 & 17.82 & 8.89 & 0.67 & 6.28 \\
\hline comp9112_c0 & 10.67 & 7.02 & 27.29 & 6.92 & 7.91 & 10.31 & 0.93 & 4.55 & 3.16 & 21.24 \\
\hline comp43544ㄱ﹎c0 & 11.83 & 2.76 & 2.87 & 3.74 & 10.29 & 16.24 & 7.80 & 7.94 & 9.83 & 26.68 \\
\hline comp17518_c0 & 99.73 & 0.00 & 0.00 & 0.01 & 0.05 & 0.01 & 0.00 & 0.00 & 0.01 & 0.18 \\
\hline comp14468_c0 & 3.58 & 3.82 & 0.92 & 8.35 & 26.43 & 3.04 & 0.72 & 0.18 & 5.58 & 47.36 \\
\hline comp12756_c1 & 4.13 & 4.90 & 45.16 & 2.55 & 9.04 & 7.08 & 1.51 & 1.01 & 9.30 & 15.32 \\
\hline comp451549_c0 & 4.51 & 1.24 & 0.55 & 0.76 & 12.44 & 7.27 & 0.99 & 0.74 & 2.97 & 68.53 \\
\hline comp10775_c0 & 3.98 & 4.82 & 2.57 & 4.35 & 10.53 & 8.30 & 4.66 & 3.82 & 9.64 & 47.33 \\
\hline \multicolumn{11}{|l|}{ Ionotropic receptor } \\
\hline comp3118_c0 & 0.76 & 0.82 & 0.48 & 1.65 & 11.96 & 0.74 & 0.25 & 0.13 & 1.03 & 82.19 \\
\hline comp2436-78_c0 & 3.25 & 1.76 & 1.29 & 3.24 & 20.10 & 5.25 & 0.79 & 2.92 & 1.65 & 59.75 \\
\hline comp369944_c0 & 0.43 & 0.45 & 0.29 & 0.43 & 21.79 & 0.99 & 0.14 & 0.26 & 0.15 & 75.07 \\
\hline comp215061_c0 & 0.15 & 0.05 & 0.11 & 0.07 & 20.15 & 0.16 & 0.03 & 0.03 & 0.10 & 79.15 \\
\hline comp264607_c0 & 11.69 & 13.68 & 7.66 & 7.32 & 7.90 & 7.32 & 6.47 & 11.30 & 3.36 & 23.29 \\
\hline comp14614_c-1 & 0.16 & 0.07 & 0.11 & 0.07 & 21.27 & 0.15 & 0.02 & 0.05 & 0.09 & 78.00 \\
\hline comp10726_c1 & 2.39 & 3.48 & 2.35 & 6.31 & 12.99 & 5.11 & 10.68 & 0.76 & 12.46 & 43.48 \\
\hline comp3333_c0 & 0.33 & 0.10 & 0.06 & 0.22 & 24.69 & 0.29 & 0.00 & 0.03 & 0.00 & 74.29 \\
\hline comp10889 & 0.78 & 1.74 & 1.27 & 37.61 & 2.68 & 2.89 & 2.31 & 36.56 & 5.45 & 8.72 \\
\hline
\end{tabular}

transcriptomic dataset. Based on antennal transcriptome analysis, 49 ORs, 2 GRs, and 15 IRs were identified in D. ponderosae and 43 ORs, 6 GRs, and 7 IRs in I. typographus (Andersson et al., 2013). The total number (36) of candidate $R$. ferrugineus chemoreceptors identified is only slightly smaller than the 66 and 56 chemoreceptors, respectively, revealed in the antennal transcriptomes of D. ponderosae and I. typographus. A greater number of $R$. ferrugineus chemoreceptors is expected to be discovered from combining specific sensory tissues and deeper transcriptome sequencing. Because major chemosensory gene families in Coleoptera have been identified only from the genome of $T$. castaneum and transcriptome of $D$. ponderosae and I. typographus, the gene sets reported here represent significant additions to the pool of the identified olfactory genes in Coleoptera (Andersson et al., 2013). As expected, T. castaneum and $D$. ponderosae are the species that returned the most BLAST hits with the $R$. ferrugineus transcriptome transcripts, because their olfactory genes currently represent the vast majority of coleopteran sequences available in GenBank. Interestingly, most of the chemoreceptor genes have a low expression level. The whole body of adults used as the sequencing samples 
other then olfactory and gustatory tissues likely account for this because olfactory and gustatory tissues represented only a very minor part of the body from which total RNA was isolated (Zhang et al., 2014). Those few chemoreceptor genes with significantly high expression levels may play important roles in the adult developmental stage.

ORs form a large and diverse family primarily responsible for the molecular recognition of olfactory stimuli in the insect environment and they are expressed on the dendrites of olfactory neurones housed within chemosensilla (Wanner et al., 2007). The transcript expression profiling of all identified OR genes in different tissues revealed that most of their expression was mainly restricted to the olfactory organs in antennae. Male-biased expression were observed in those mainly expressed in antennae. Very low expression in the female antennae of ORs such as comp2310_c0, comp178288_c0, comp40965_c0, comp11894_c0, and comp12077_c0 suggests that they may mediate an olfactory behavior mainly specific to males. The findings that all receptor types hitherto identified are predominantly expressed in the antennae of males and females suggests that they may be involved in the detection of general odors rather than of pheromones (Krieger et al., 2002). Thus, they may be the candidate receptors for semiochemicals from females or the infested hosts and used to find emerging female adults (Ma et al., 2014). They may be also essential for this insect to find its host plants. Three genes (comp41421_c0, comp186697_c0, and comp1452_c0) are expressed at high levels in legs. The transcript levels of comp41421 c0 and comp1452 c0 are higher in female legs than in male legs. In addition, comp14522_c0 appeared to be enriched in the abdomen, and comp12012_c0 was expressed at higher level in the abdomen than in antennae. It is unlikely that proteins predominantly expressed in non-olfactory tissues play a role in odorant reception in antennae (Leal et al., 2013). These OR genes are enriched in non-olfactory tissues, suggesting that they may play an important role in the unexpected expression in non-chemosensory tissues. Insect GRs, which are unrelated to mammalian taste receptors, are now known to function in the detection of sugars, bitter compounds, and nonvolatile pheromones by reorganizing gustatory stimuli from the environment (Freeman et al., 2014). GRs are generally expressed in GR neurons. With the exception of the antennal location, some of the identified GRs are highly expressed in the head, thorax, and abdomen. This is consistent in that gustatory sensilla are not restricted to the region around the mouth but are usually distributed over different regions of the body surface and appendages such as the antennae, mouth parts, leg tarsi, and margins of the wings (de Brito Sanchez et al., 2014). In particular, comp17518_c0 was predominantly enriched in female heads. It may perform the appropriate physiological functions that fit its sexual location. In all testis tissues, comp10251_c0, comp435449_c0, comp451549_c0, and comp10775_c0 showed more than a two-fold sexual biased expression, and they may be involved in mediating sex-specific behaviors. IRs were recently identified as a novel group of chemosensory receptors, first in Drosophila melanogaster and then in several other species, hinting at a broader function in detection of environmental as well as intercellular chemical signals (Croset et al., 2010; Liu et al., 2012). In addition to comp264607_c0 and comp10889_c0, the other seven identified IRs, like ORs and GRs, were predominantly located in antennae. In D. melanogaster, 15 of the total 66 IRs were shown to be specifically expressed in antennae, where they localize in the dendrites of olfactory sensory neurons housed in coeloconic sensilla (Benton et al., 2009). Comp264607_c0 and comp10889_c0 appeared to be expressed in the head, thorax, abdomen, and leg. Some of these tissues are known to carry contact chemosensory sensilla (Widmayer et al., 2009). Furthermore, studies observed that some IR transcripts were not specific to chemosensory tissues, indicating such IRs may be 
involved in functions other than chemoreception (Olivier et al., 2011). The expression patterns of chemorecptors in R. ferrugineus may help guide electrophysiological and chemical ecology studies for characterizing their function in future research.

\section{Conflicts of interest}

The authors declare no conflict of interest.

\section{ACKNOWLEDGMENTS}

Research supported by the Key Program for Science and Technology and the Major projects of Hainan, China (\#ZDXM20130049 and \#ZDZX2013008) and the Scientific Research Project of CATAS (\#1630042014002).

\section{Supplementary material}

\section{REFERENCES}

Abraham VA, Faleiro JR, Kumar TP and Al-Shuaibi MA (1999). Sex ratio of red palm weevil Rhynchophorus ferrugineus Olivier captured from date plantation of Saudi Arabia using pheromone traps. Indian J. Entomol. 16: 201-204.

Abuagla AM and Al-Deeb MA (2012). Effect of bait quantity and trap color on the trapping efficacy of the pheromone trap for the red palm weevil, Rhynchophorus ferrugineus. J. Insect Sci. 12: 120.

Andersson MN, Grosse-Wilde E, Keeling CI, Bengtsson JM, et al. (2013). Antennal transcriptome analysis of the chemosensory gene families in the tree killing bark beetles, Ips typographus and Dendroctonus ponderosae (Coleoptera: Curculionidae: Scolytinae). BMC Genomics 14: 198.

Avalos JA, Martí-Campoy A and Soto A (2014). Study of the flying ability of Rhynchophorus ferrugineus (Coleoptera: Dryophthoridae) adults using a computer-monitored flight mill. Bull. Entomol. Res. 104: 462-470.

Barranco P, De la Penã J and Cabello T (1997). Cría artificial de Rhynchophorus ferrugineus (Olivier, 1790) (Col.: Curculionidae). In: Resúmenes IV Congreso Nacional de la Sociedad Espaňola para las Ciencias del Animal de Laboratorio. Vitoria, CC-03.

Benton R, Vannice KS, Gomez-Diaz C and Vosshall LB (2009). Variant ionotropic glutamate receptors as chemosensory receptors in Drosophila. Cell 136: 149-162.

Conesa A, Götz S, García-Gómez JM, Terol J, et al. (2005). Blast2GO: a universal tool for annotation, visualization and analysis in functional genomics research. Bioinformatics 21: 3674-3676.

Croset V, Rytz R, Cummins SF, Budd A, et al. (2010). Ancient protostome origin of chemosensory ionotropic glutamate receptors and the evolution of insect taste and olfaction. PLoS Genet. 6: e1001064.

de Brito Sanchez MG, Lorenzo E, Su S, Liu F, et al. (2014). The tarsal taste of honey bees: behavioral and electrophysiological analyses. Front. Behav. Neurosci. 8: 25.

Engsontia P, Sanderson AP, Cobb M, Walden KK, et al. (2008). The red flour beetle's large nose: an expanded odorant receptor gene family in Tribolium castaneum. Insect Biochem. Mol. Biol. 38: 387-397.

Faleiro JR and Ashok Kumar J (2008). A rapid decision sampling plan for implementing area-wide management of the red palm weevil, Rhynchophorus ferrugineus, in coconut plantations of India. J. Insect Sci. 8: 15.

Freeman EG, Wisotsky Z and Dahanukar A (2014). Detection of sweet tastants by a conserved group of insect gustatory receptors. Proc. Natl. Acad. Sci. U. S. A. 111: 1598-1603.

Glaser N, Gallot A, Legeai F, Montagné N, et al. (2013). Candidate chemosensory genes in the Stemborer Sesamia nonagrioides. Int. J. Biol. Sci. 9: 481-495.

Grabherr MG, Haas BJ, Yassour M, Levin JZ, et al. (2011). Full length transcriptome assembly from RNA-Seq data without a reference genome. Nat. Biotechnol. 29: 644-652.

Krieger J, Raming K, Dewer YM, Bette S, et al. (2002). A divergent gene family encoding candidate olfactory receptors of the moth Heliothis virescens. Eur. J. Neurosci. 16: 619-628.

Leal WS, Choo YM, Xu P, da Silva CS, et al. (2013). Differential expression of olfactory genes in the southern house mosquito and insights into unique odorant receptor gene isoforms. Proc. Natl. Acad. Sci. U. S. A. 110: 18704-18709. 
Liu Y, Gu S, Zhang Y, Guo Y, et al. (2012). Candidate olfaction genes identified within the Helicoverpa armigera antennal transcriptome. PLoS One 7: e48260.

Livak KJ and Schmittgen TD (2001). Analysis of relative gene expression data using real-time quantitative PCR and the $2^{-\Delta \Delta C t}$ method. Methods 25: 402-408.

Llácer E, Santiago-Álvarez C and Jacas JA. (2013). Could sterile males be used to vector a microbiological control agent? The case of Rhynchophorus ferrugineus and Beauveria bassiana. Bull. Entomol. Res. 103: 241-250.

Ma L, Gu SH, Liu ZW, Wang SN, et al. (2014). Molecular characterization and expression profiles of olfactory receptor genes in the parasitic wasp, Microplitis mediator (Hymenoptera: Braconidae). J. Insect Physiol. 60: 118-126.

Mortazavi A, Williams BA, McCue K, Schaeffer L, et al. (2008). Mapping and quantifying mammalian transcriptomes by RNA-Seq. Nat. Methods 5: 621-628.

Olivier V, Monsempes C, François MC, Poivet E, et al. (2011). Candidate chemosensory ionotropic receptors in a Lepidoptera. Insect Mol. Biol. 20: 189-199.

Poivet E, Gallot A, Montagné N, Glaser N, et al. (2013). A comparison of the olfactory gene repertoires of adults and larvae in the noctuid moth Spodoptera littoralis. PLoS One 8: e60263.

Sánchez-Gracia A1, Vieira FG and Rozas J (2009). Molecular evolution of the major chemosensory gene families in insects. Heredity Edinb103: 208-216.

Vieira FG and Rozas J (2011). Comparative genomics of the odorant-binding and chemosensory protein gene families across the Arthropoda: origin and evolutionary history of the chemosensory system. Genome Biol. Evol. 3: 476-490.

Wanner KW, Anderson AR, Trowell SC, Theilmann DA, et al. (2007). Female-biased expression of odourant receptor genes in the adult antennae of the silkworm, Bombyx mori. Insect Mol. Biol. 16: 107-119.

Widmayer P, Heifetz Y and Breer H. (2009). Expression of a pheromone receptor in ovipositor sensilla of the female moth (Heliothis virescens). Insect Mol. Biol. 18: 541-547.

Zhang Y, Zheng Y, Li D and Fan Y (2014). Transcriptomics and identification of the chemoreceptor superfamily of the pupal parasitoid of the oriental fruit fly, Spalangia endius Walker (Hymenoptera: Pteromalidae). PLoS One 9: e87800.

Zhou SS, Sun Z, Ma W, Chen W, et al. (2013). De novo analysis of the Nilaparvata lugens (Stål) antenna transcriptome and expression patterns of olfactory genes. Comp. Biochem. Physiol. Part D Genomics Proteomics 9: 31-39.

Zhu JY, Zhao N and Yang B (2012). Global transcriptional analysis of olfactory genes in the head of pine shoot beetle, Tomicus yunnanensis. Comp. Funct. Genomics Article ID 491748.

Zhu JY, Yang P, Zhang Z, Wu GX, et al. (2013). Transcriptomic immune response of Tenebrio molitor pupae to parasitization by Scleroderma guani. PLoS One 8: e54411. 